\title{
Caracterización Socioeconómica de la Comunidad Indígena Wayuu de Manzana en Colombia: Un aporte desde la Ingeniería Social
}

\author{
Marlin A. Aarón ${ }^{(1)^{*}}$, Andrés D. Solano(1), Patricia E. Choles ${ }^{(1)}$ y Rosalba Cuesta ${ }^{(1)}$ \\ (1) Universidad de La Guajira, Facultad de Ingenierías, Educación y Ciencias Económicas y administrativas. \\ Grupo Investigación y Desarrollo Motivar. Riohacha, Km 5 Salida a Maicao, La Guajira-Colombia. \\ (e-mail: maaron@uniguajira.edu.co; andresolano@uniguajira.edu.co; pcholes@uniguajira.edu.co; \\ rcuestas@uniguajira.edu.co)
}

* Autor a quien debe ser dirigida la correspondencia

Recibido Feb. 28, 2018; Aceptado May. 9, 2018; Versión final Jul. 10, 2018, Publicado Dic. 2018

\begin{abstract}
Resumen
Se presenta un análisis sobre las condiciones socioeconómicas de la comunidad indígena Wayuu de Manzana ubicada en el municipio de Manaure, departamento de La Guajira, Colombia. El estudio se realizó enfocado desde la Ingeniería Social, entendida como la posibilidad que tiene el estudiante de ingeniería de visibilizar la realidad, no solo desde datos numéricos sino aproximándose a ella. La metodología utilizada en esta investigación es de tipo cuali-cuantitativa, usando la estadística para registrar datos, contar, seleccionar y categorizar aspectos que permiten comprender y explicar la realidad. Lo cualitativo se obtiene a partir de la observación directa, la descripción y la representación gráfica. Esta permitió contrastar la realidad que percibían los sentidos y los resultados estadísticos. Se utilizó como herramienta de trabajo una encuesta aplicada a 484 hogares de la comunidad. Uno de los factores que impiden el desarrollo de la comunidad es la poca comprensión entre la interacción de los problemas tecnológicos, sociales y la apropiación de la realidad. Se logró que los estudiantes reconocieran el valor del análisis del contexto más allá de los resultados numéricos.
\end{abstract}

Palabras clave: ingeniería social; caracterización; comunidad indígena Wayuu; condición socioeconómica.

\section{Socioeconomic Characterization of the Wayuu Manzana Indigenous Community in Colombia: A Contribution from Social Engineering}

\begin{abstract}
An analysis is presented on the socioeconomic conditions of the Wayuu indigenous community of Manzana, located in the municipality of Manaure, department of La Guajira, Colombia. The study was done from the Social Engineering concept, considered as the possibility that engineering student could visualize real life, not only from numerical data but also approaching such a reality. The methodology used in this research is qualitative-quantitative, in which statistics is used to record data, to count, to select and to categorize aspects that allow understanding and explaining reality. The qualitative aspects are collected from direct observation, description and graphic representation. All this allows contrasting the reality that perceived their senses and the statistical results. A survey was applied to 484 households in the community. One of the factors that stop development of the community is the limited understanding between the interaction of technological and social problems and the appropriation of reality. Students were able to recognize the value of context analysis beyond only numerical results.
\end{abstract}

Keywords: social engineering; characterization; Wayuu indigenous community; socioeconomic status. 


\section{INTRODUCCIÓN}

La población mundial indígena tiene una composición que representa el 5.5\%. A 2006, los cálculos dan cuenta de 370 millones de indígenas en el mundo, representados en al menos 5.000 grupos lingüísticos distintos en más de 70 países (UNESCO 2003, Evaluación de Ecosistemas del Milenio, 2005, Fundación pro Naciones Unidas-UNFII, 2007). Aunque los pueblos indígenas representan a menudo las poblaciones minoritarias de los distintos países, constituyen el 70-80 por ciento, aproximadamente, de la diversidad cultural mundial (IUCN, siglas en inglés de la Comisión de Políticas Ambientales, Económicas y Sociales, 1997).

Según (UNICEF, 2007) la población indígena total de Colombia que reporta el Departamento Nacional de Planeación (DNP), es de 1.378.884 habitantes, corresponden al 3,4\% de la población del país (DANE, Censo 2005) distribuidos en cinco macroregiones identificadas como: Amazonía, con 83 entidades territoriales (resguardos y reservas), 44 grupos étnicos y una población de 48.622, equivalente al $6.9 \%$; Orinoquia, con 124 entes territoriales, 56 grupos étnicos y 69.866 habitantes que representan el $9.9 \%$ de la población indígena nacional, en la que se destacan los Sicuani, con el $29.4 \%$, los Curripacos, con el $10.1 \%$ y los Tukano, con el $9.7 \%$ de la región. La región Centro Oriente, con 28 resguardos, entre los que se desatacan los Pijao, con el $9 \%$, los uva, con el 19\% de una población de 36.017 habitantes de la región, equivalente al $5.1 \%$ de la población indígena nacional. La región de Occidente con 222 resguardos, 27 pueblos indígenas y 337.636 habitantes, lo que representa la mayor población del país (48.1\%), destacándose el pueblo nasa o Paez, con el $35.1 \%$ de la población indígena de la región, los Embera, con 1.1\% de la región, los pastos, con el 16.4\% y los Guambianos, con el 6.1\%. La Costa Atlántica cuenta con 22 entes territoriales, 17 grupos étnicos y 209.719 habitantes que representan el $29.4 \%$ de la población de la región. El grupo mayoritario de la región es el Wayuu, con el $68.6 \%$ de la región, le siguen los Zenú, con el $16.1 \%$ y los Arhuacos, con el $6.8 \%$. . Estos pueblos indígenas identificados plenamente hablan 64 lenguas amerindias y una diversidad de dialectos que se agrupan en 13 familias lingüísticas

La comunidad indígena Wayuu habita en la árida península de La Guajira al norte de Colombia y noroeste de Venezuela sobre el Caribe, a $180 \mathrm{~m}$ sobre el nivel del mar (Ortiz et al., 2015). El Departamento Administrativo Nacional de Estadística (DANE) reportó en 2005 que 270.413 personas se reconocen como pertenecientes a la comunidad Wayuu, cifra que posiciona a esta comunidad indígena como la de mayor población en el país. El $49 \%$ de esta población son hombres (132.180) y el 51\%, mujeres (138.233). En el departamento de La Guajira Colombiana está el municipio de Manaure que tiene una extensión territorial de $1.643 \mathrm{Km}^{2}$ y está compuesto por su casco urbano y nueve corregimientos: Aremasain, El Pájaro, La Gloria, La Paz, Manzana, Mayapo, Musichi, San Antonio de Pancho y Shiruria. En el corregimiento de El Pájaro existen las mayores reservas de gas probadas del país en los campos de Chuchupa I y II, Ballenas y Estación Riohacha. La explotación de gas en la Guajira está siendo llevada a cabo por la empresa extranjera Chevron Petroleum Company, según el contrato de asociación Guajira "A", celebrado con EcopetroL (Empresa colombiana de Petróleos), en un área total de 81,974 Hectáreas. Esta caracterización se realizó en una (1) comunidad indígena Wayuu del Municipio de Manaure, ubicada en Manzana, que está geográficamente cercana al lugar donde se realiza la explotación del gas, lo que implica responsabilidad social por parte de la empresa operadora.

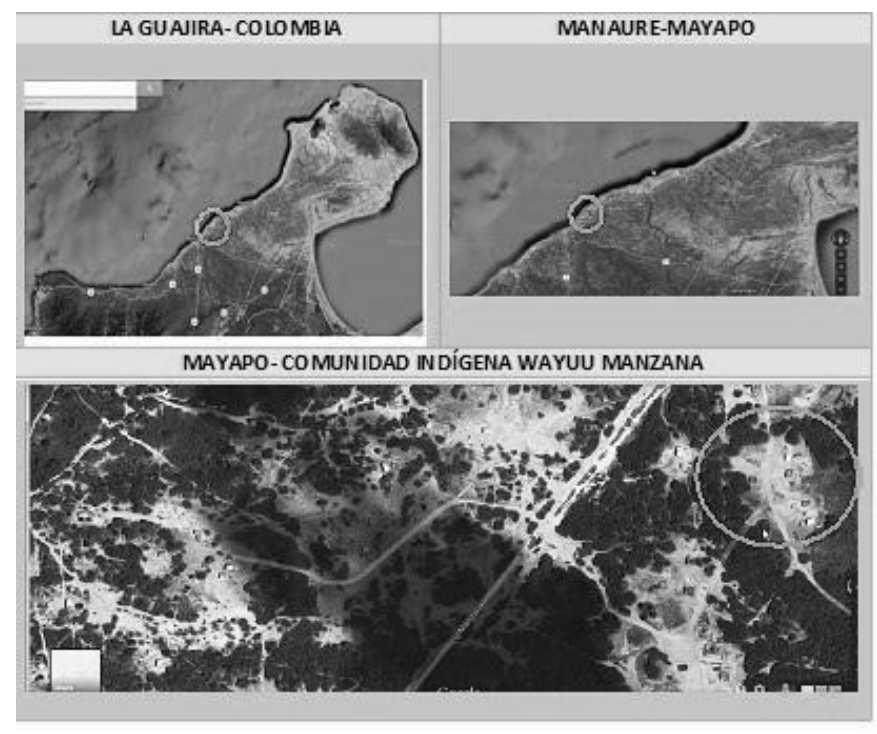

Fig. 1. Imagen tomada de Google earth modificada por los autores, Que muestra la ubicación de comunidad indígena Wayuu de Manzana. 
Según la legislación colombiana, las "comunidades indígenas" son el grupo humano que vive de acuerdo con las formas de relación con el medio natural en el que se asentaron los diferentes grupos aborígenes desde antes de la conquista y la han conservado y dinamizado a lo largo de la historia. Un pueblo indígena puede estar compuesto por varias comunidades, es decir, una comunidad indígena constituye un fragmento de un pueblo indígena (Dussán y Rodriguez, 2005). Según el Decreto 2164 de 1995, por el cual se reglamenta parcialmente el capítulo XIV de la ley 160 de 1994, en su artículo 2, (Rodríguez, 2017) define la Comunidad o Parcialidad Indígena, como el grupo o conjunto de familias, de ascendencia amerindia, que tienen conciencia de identidad y comparten valores, rasgos, usos o costumbres de su cultura, así como formas de gobierno, gestión, control social o sistemas normativos propios que la distinguen de otras comunidades, tengan o no títulos de propiedad, o que no puedan acreditarlos legalmente o que sus resguardos fueron disueltos, divididos o declarados vacantes. Esta considerable población necesita ser identificada no solo desde sus aspectos de ubicación geográfica y lengua, sino conocer cuáles son las condiciones en que viven y sus relaciones con el entorno. En términos económicos, los pueblos indígenas están desproporcionadamente representados entre los pobres y respecto a la inseguridad alimentaria, tanto en los países desarrollados como en los en vías de desarrollo (Psacharopoulos y Patrinos, 1994; Carino, 2005; UNPFII, 2005). En la mayoría de los países del mundo son evidentes las condiciones de pobreza en que viven, independientemente de la ubicación geográfica o su cosmovisión. La comunidad indígena Wayuu de Manzana (Sea, 2012) (Premauer y Berkes, 2015) hace parte de las comunidades indígenas que habitan en La Guajira, Colombia, y además pertenece a la zona de influencia de la Universidad de La Guajira, quien desarrolla este proyecto de investigación, por ello, cómo parte de su función de docencia, investigación y de proyección social debe reconocer como son los escenarios y los aspectos que caracterizan este tipo de comunidades.

Este documento se refiere a condiciones socioeconómicas de la comunidad indígena Wayuu de Manzana. Hall y Patrinos indican que no hay duda que los pueblos indígenas se relacionan con bajo nivel de educación, condiciones deficientes en términos de nutrición, salud, desempleo y discriminación, así como otros temas que se abordan como elementos constitutivos de la "pobreza". Sin embargo, los pueblos indígenas también se consideran a sí mismos ricos en materia de tradiciones culturales y espirituales, a las que la sociedad en general suele asignar mucho menos valor y que no se prestan para mediciones cuantitativas (Hall y Patrinos, 2005). Sen señala el concepto de pobreza (Desai, 2017) a partir de las capacidades, es decir lo que la gente puede hacer, definiendo la pobreza como la ausencia de capacidades básicas que le permiten a cualquier individuo insertarse en la sociedad, a través del ejercicio de su voluntad. En un sentido más amplio, la pobreza no es cuestión de escaso bienestar, sino de incapacidad de conseguir bienestar precisamente debido a la ausencia de medios. En resumen Sen afirma que la pobreza no es falta de riqueza o ingreso, sino de capacidades básicas (Sen, 1992). Según Cortés (2002), la marginación en su versión más abstracta intenta dar cuenta del acceso diferencial de la población al disfrute de los beneficios del desarrollo. La medición se concentra en las carencias de la población de las localidades en el acceso a los bienes y servicios básicos, captados en tres dimensiones: educación, vivienda e ingresos". Para él, la marginación es un fenómeno que afecta a las localidades y no necesariamente a las personas que viven en ellas.

La ingeniería social, concepto este que aún requiere más documentación y divulgación, es presentado por Galindo ( 2010) como la aplicación de conocimiento específico de lo social, sobre lo social, y para lo social, a la invención, perfeccionamiento y utilización de reglas prácticas para construir formas de compañía, de asociación y comunidad. Una perspectiva amplia que considera cualquier tipo de conocimiento social como utilizable para la construcción de relaciones entre los individuos y los grupos que buscan convivir en un tiempoespacio. Lo cual se complementa con la configuración de operaciones para formar, fomentar, evolucionar, enriquecer, la vida en sociedad en general, de compañía en particular. El énfasis está en la relación entre el conocimiento social y las formas técnicas de construcción de la convivencia en sociedad (Kennedy y Parsons, 2012). Otra definición más específica podría ser aplicación de principios científicos al diseño y construcción de formas de asociación y comunidad en grupos organizados. Lo cual implica la creación de formas de asociación, de seguimiento, del contacto, la interacción, el vínculo, la conexión y la comunicación. Para este estudio, fue considerada como una estrategia de enseñanza de análisis de contexto (Aarón, 2016) para la contrastación entre la representación de la realidad con la misma realidad, percibida desde los sentidos y la forma como esto puede servir para provocar en el ser humano que observa unas nuevas formas de proveer soluciones a la problemática que observa. Esta estrategia fue aplicada a los estudiantes que participaron como encuestadores en la comunidad indígena Wayuu de Manzana.

Desde una perspectiva investigativa la caracterización es una fase descriptiva con fines de identificación, entre otros aspectos de los componentes, acontecimientos (cronología e hitos), actores, procesos y contexto de una experiencia, un hecho o un proceso Sánchez (2010). La caracterización es un tipo de descripción cualitativa (Chenail, 2012; Creswell 2019) que puede recurrir a datos o a lo cuantitativo con el fin de profundizar el conocimiento (Olivares, et al., 2017) sobre algo. Para cualificar ese algo previamente se deben identificar y organizar los datos; y a partir de ellos, describir (caracterizar) de una forma estructurada; y posteriormente, establecer su significado (sistematizar de forma crítica) (Bonilla et al., 2009). 
Se realiza este caracterización como un subproyecto de investigación, dentro del Proyecto de investigación, innovación y desarrollo, denominado "Incorporación de las tecnologías móviles para potenciar los estilos de aprendizajes en la comunidad educativa del área de influencia de la Universidad de La Guajira: caso específico: estudiantes indígenas Wayuu de 5to grado de la Comunidad de Manzana" el que se estructura por fases formativas, a partir de metodología cualitativa, desde la Investigación-acción-participación AnderEgg (2003) las que en su fase inicial identifican las condiciones en que se encuentran, y las necesidades básicas de las comunidades educativas indígenas de la etnia Wayuu. Los resultados de la investigación sobre la caracterización socioeconómica que se presentan en este artículo, sirven como aporte para ese macroproyecto.

El análisis se consolida a partir de encuestas aplicadas a miembros de la comunidad indígena Wayuu de Manzana. Se desarrolló durante 6 meses(febrero, agosto de 2015), realizado con expertos en estadística y apoyado con estudiantes del programa de Ingeniería de Sistemas, donde por medio de la Ingeniería Social, se logra el acercamiento de estudiantes a la comunidad indígena, con la intención de observar la complejidad de estas, - en este caso, además de indígena, en zona rural-, tal como lo expresa López (2015), quién establece un principio de la Ingeniería Social, donde explica que el ser humano no puede concebir la complejidad de los problemas; por ello necesita construir nuevas formas de intervenir en el mundo, desde la práctica y desde la ciencia. Así mismo, García (2012) afirma que la construcción de cultura de participación y la Ingeniería Social tienen una estrecha relación, que permite dar solución a problemas sociales.

El objetivo de incorporar a los estudiantes en este proceso de caracterización socioeconómica de la comunidad indígena dentro de una línea de investigación de Ingeniería Social en el programa de Ingeniería de Sistemas, responde a desarrollar trabajos investigativos orientados en el diseño y la gestión de productos y proyectos tecnológicos con una perspectiva contextual, transversal e interdisciplinaria, con habilidades para comprender la interacción entre problemas tecnológicos y sociales, la responsabilidad social y los procesos de transformación tecnológica. Hay un interés expreso en la enseñanza de la asignatura de Modelos a la que pertenecen los estudiantes, en que estos realicen un reconocimiento desde la ingeniería social y pasen del conocimiento tácito al explicito Aarón et al., (2014); lo que creían que pasaba en la comunidad indígena, con lo que realmente pasa en la comunidad indígena, percibido desde sus sentidos, y registrado a través de datos, pero también desde la representación descriptiva y gráfica, usando diagramas, centrando la importancia en como la ingeniería en estos escenarios, puede servir para aportar soluciones.

\section{METODOLOGÍA}

La metodología utilizada en esta investigación es descriptiva de tipo cualicuantitativa, apoyada por la estadística para la descripción, comprensión y explicación de la realidad de esta comunidad. Lo cualitativo, permitió a los estudiantes, -quienes participan como encuestadores-, desde la observación directa y la representación gráfica, contrastar si la realidad que percibían sus sentidos y los resultados estadísticos, eran iguales. Identificar en situ, si el modelo descriptivo, representado en números, era capaz de representar en su totalidad la realidad. Para la obtención de la información se utilizó como herramienta de trabajo una encuesta aplicada a 484 hogares, para una población total de 2.020 personas, en la Comunidad indígena Wayuu de Manzana, Mayapo municipio de Manaure, departamento de la Guajira, Colombia, la cual fue seleccionada por ser de área de influencia de La Universidad de La Guajira. Esto corresponde al universo de la población.

Dicha encuesta fue estructurada en veinticuatro preguntas subdivididas en secciones: características generales de la población y datos de la forma como viven. Se recoge información general del jefe de hogar: nombre, apellido, edad, sexo, estado civil y nivel de escolaridad actual, lenguas que habla, wayunaiki y español. Las preguntas sobre datos de la vivienda y actividad económica del jefe de hogar, si se da el suministro del agua a la vivienda y cómo, si cuenta con servicio de energía, tratamiento de la basura, servicio sanitario, aparatos de uso doméstico, material de las paredes exteriores, del techo y del piso y nivel educativo de los miembros de la familia.

Después de recolectada la información, se descargó al formato montado en el software SPSS, para su respectivo análisis. Los estudiantes participaron en la recolección de la información, encuestando y percibiendo de manera directa la realidad que estaban registrando. En cada sesión de visitas (6) a la Comunidad para realizar las encuestas, los estudiantes diligenciaban un formato de observación, en el que las preguntas estaban centradas en lo que habían visto y percibido en cada sesión y lo que más les había impactado, así como la forma como ellos explicaban intuitivamente aquellas situaciones que habían observado y habían sido de impacto. Al final todos estos registros fueron consolidados y se realizó una descripción de todo lo observado, así como las explicaciones que ellos daban a los sucesos. Luego se contrastó con los datos obtenidos estadísticamente y se hacían sesiones de análisis sobre ambos resultados. 
Se generó un puente entre la metodología de investigación cualicuantitativa y la estrategia de enseñanza soportada en la ingeniería social, para posibilitar la presencia de los estudiantes como encuestadores y observadores de la realidad. El producto de la investigación que corresponde a las condiciones socioeconómicas, además de datos y frecuencias, se convierte en insumo para la contrastación de la realidad desde la forma como esos datos, son percibidos por quienes los recogen.

\section{RESULTADOS Y DISCUSIÓN}

La aplicación del instrumento diseñado suministra información que permite identificar las características más relevantes de los habitantes de Manzana. Se caracterizó el universo de la población, 484 hogares, para una población total de 2.020 personas, de las cuales un 54\% de la población habla dos lenguas (español y Wayunaiki), el $45 \%$ de la población solo habla Wayunaiki y el 1\% de la población habla únicamente español. Resultados sobre el jefe del hogar se pueden detallar en la tabla 1:

Tabla 1: El jefe de hogar en comunidad Indígena Wayuu de Manzana.

\begin{tabular}{c|c|c}
\hline Variable & Categorías & Frecuencia (\%) \\
\hline Sexo del jefe cabeza hogar. & Masculino & 33 \\
\hline Edad del Jefe cabeza de hogar & Femenino & 67 \\
\hline Estado civil de los Jefes Cabeza de Hogar & $10-20$ & 29 \\
& 30 a 40 & 26 \\
& 20 a 30 & 16 \\
& 40 a 50 & 9 \\
& 50 a 60 & 11 \\
\hline Nivel educativo del Jefe cabeza de hogar & Unión Libre & 68 \\
& Separados & 13 \\
& Solteros & 8 \\
& Vin nivel de escolaridad & 6 \\
& Primaria Incompleta & 4 \\
& Secundaria incompleta & 1 \\
\hline & Primaria Completa & 52 \\
& Secundaria Completa & 29 \\
\hline
\end{tabular}

El análisis de los resultados de la investigación, permite visualizar características socioeconómicas de los pobladores de la comunidad de Manzana. Los jefes de hogar están representados en un $67 \%$ por las mujeres y un $33 \%$ son del sexo masculino, como se muestra en la tabla 1. La edad predominante de ellos, está en el grupo etario de 30 a 40 años con una participación porcentual del $29 \%$, seguido por el grupo de 20 a 30 años con un 26\%; los de 40 a 50 años representan un 16\%. Los mayores de 60 años tienen una participación 11\%, mientras que los grupos etarios de 10 a 20 años y de 50 a 60 años, aportan una participación del 9\%.

Con respecto al el estado civil de los jefes cabeza de hogar de la comunidad de Manzana, predomina la unión libre con un $68 \%$ de participación, seguido de los separados con una participación del $13 \%$, siendo los solteros el $8 \%$, para una participación total del $89 \%$, mientras que el $11 \%$ restante corresponden a viudos, casados y divorciados.

En cuanto al nivel educativo de los jefes cabeza de hogar, este es bajo. La mayoría no ha ingresado a ningún centro educativo y los que lo han hecho han desertado. Un $52 \%$ no ha realizado estudios, seguido por un $29 \%$ con primaria incompleta, un $8,7 \%$ con secundaria incompleta, el $4 \%$ tiene primaria completa, el $3,3 \%$ tiene secundaria completa, un 1,4\% tiene formación universitaria Incompleta y el 1\% restante, tiene formación técnica. 
Tabla 2: Servicios públicos domiciliarios Comunidad Indígena Wayuu de Manzana

\begin{tabular}{c|c|c}
\hline Variable & Categorías & Frecuencia (\%) \\
\hline $\begin{array}{c}\text { Servicios públicos } \\
\text { domiciliarios }\end{array}$ & Red pública de energía eléctrica & 79 \\
& Sin servicio de energía. & 20 \\
Planta eléctrica propia & 1 \\
& Sin servicio sanitario & 82 \\
& Tubería conectada a pozos & 18 \\
& sépticos & \\
& Sin servicio de Aseo & 100 \\
\hline
\end{tabular}

Se muestra en la tabla 2, que en general los servicios públicos son deficientes. El servicio de alcantarillado con bajísima cobertura, el $82 \%$ de la población no tiene servicio sanitario y el $18 \%$ tiene tubería conectada a pozos sépticos; esta situación es preocupante porque los habitantes tienen que hacer sus necesidades en cualquier parte, convirtiéndose en focos de infección para la comunidad. El servicio de aseo no existe, por tal motivo el $81 \%$ de los hogares queman la basura, un $2 \%$ la entierra y el resto $17 \%$, la tiran en cualquier parte o en lotes baldíos; al quemar la basura se contamina el medio ambiente, afecta la salud por la inhalación de humo y al arrojarla a cualquier parte se convierte en contaminación visual y una amenaza para la misma población. Sumado a lo anterior, el servicio de energía eléctrica es deficiente; el $79 \%$ de la población está conectado a red pública, el $20 \%$ no tiene servicio de energía y el $1 \%$ tiene red propia; el servicio de energía en términos generales presenta debilidades por el bajo voltaje y cortes permanentes, con conexiones que no cumplen las normas, lo cual representa un riesgo para la comunidad, lo que es óbice para el uso de aparatos que se alimentan de energía eléctrica. En la tabla 3, se presentan los datos con respecto al uso de estos aparatos.

Tabla 3: Aparatos de uso doméstico comunidad Indígena Wayuu de Manzana

\begin{tabular}{c|c|c}
\hline Variable & Categorías & Frecuencia (\%) \\
\hline Aparatos de uso & Radio & 33,8 \\
doméstico & Televisor & 23,7 \\
& Teléfono móvil & 18,4 \\
& Licuadora & 8,9 \\
& Nevera o enfriador & 7,1 \\
& Equipo de sonido & 6.7 \\
& Lavadora & 1,0 \\
& Computador & 0,40 \\
\hline
\end{tabular}

Lo que más usan los hogares es la radio, con un promedio del $33,8 \%$, seguido del televisor con un $23,7 \%$, un bajo uso de telefonía móvil, el 18,4\%, un 8,9\% la licuadora. La nevera o enfriador con un $7,1 \%$ y equipo de sonido en un $6.7 \%$ siendo estos aparatos con porcentajes más significativos; solo el 1,04\% utiliza lavadora y computador.

Tabla 4: Actividad económica Comunidad Indígena Wayuu de Manzana.

\begin{tabular}{c|l|l}
\hline \multicolumn{1}{c|}{ Variable } & \multicolumn{1}{|c}{ Categorías } & \multicolumn{1}{c}{ Frecuencia (\%) } \\
\hline Actividad económica & Artesanía & 40 \\
& Pesca & 34 \\
& Pastoreo & 22 \\
& Turismo, cultivos agrícolas y otras & 4 \\
\hline
\end{tabular}

Las actividades económicas son desarrolladas por el núcleo familiar. Las principales, se pueden observar en la tabla 4. No demandan formación en centros educativos para el desarrollo de ellas, ya que los oficios que desarrollan lo aprenden en el hogar y hacen parte de su cosmovisión y cultura. La de mayor porcentaje es la artesanía que representa el $40 \%$, seguida por la pesca y el pastoreo, siendo estas las más representativas con un porcentaje acumulado del $96 \%$; el $4 \%$ restante está entre turismo, cultivos agrícolas y otras.

En la tabla 5, se presentan los datos correspondientes a la formación académica de la comunidad. Su bajo nivel educativo evidencia características que dificultan obtener buenas condiciones para su pervivencia. A través de la educación se identifica el grado de desarrollo de las comunidades, pueblos o naciones, de tal modo que se establecen diferencias entre los países desarrollados y los hoy de manera eufemística 
denominados emergentes, ello obedece al factor educación los cuales son totalmente diferentes en calidad y eficiencia y efectividad. De igual modo entre las comunidades de un mismo país, se presentan diferentes niveles de calidad y cobertura en la educación de su talento humano, a pesar de disponer de una misma legislación educativa, lo cual va a impactar de manera directa en el desarrollo de su economía, del sector productivo y de las condiciones sociales de sus habitantes. En lo relacionado con la educación de la población total de la comunidad indígena Wayuu de manzana, se encontró que el 41,5\% de los habitantes de la comunidad de Manzana su primaria es incompleta, el 34\% no ha realizado ningún estudio; solo un 13\% cuenta con la secundaria completa, el 5,6\% primaria completa y el 1\% restante tiene educación nivel técnica. Los datos de esta comunidad preocupan porque el nivel del analfabetismo es del 31\%, donde el promedio nacional está en un $8 \%$, lo que muestra una sensible desigualdad. Es alto el porcentaje de la población que inició estudios y desertó, lo que repercute en el desarrollo de la comunidad.

Tabla 5: Formación Académica población total Comunidad Indígena Wayuu de Manzana

\begin{tabular}{l|l|l}
\hline \multicolumn{1}{c|}{ Variable } & \multicolumn{1}{|c}{ Categorías } & Frecuencia (\%) \\
\hline Formación académica población total & Primaria incompleta & 41,5 \\
& Primaria completa & 5,6 \\
& Sin ningún estudio & 34 \\
& Secundaria Completa & 13 \\
& Secundaria Incompleta & 3,5 \\
& Universitaria & 1,4 \\
& Técnica & 1 \\
\hline
\end{tabular}

Tabla 6: Suministro de agua en la Comunidad Indígena Wayuu de Manzana.

\begin{tabular}{c|l|l}
\hline \multicolumn{1}{c|}{ Variable } & \multicolumn{1}{|c}{ Categorías } & \multicolumn{1}{|c}{ Frecuencia (\%) } \\
\hline Suministro de agua & Molinos de Viento & 69 \\
& Pozos con tuberías o bombas & 28 \\
& Camiones Cisternas & 2 \\
& Agua de Jagüeyes & 1 \\
\hline
\end{tabular}

Con relación al suministro de agua en sus viviendas, la tabla 6 muestra que el $69 \%$ de las viviendas la reciben de pozos con molinos de viento, un $28 \%$ de pozos con tuberías o bombas, un $2 \%$ camiones cisternas y el $1 \%$ restante toma agua de Jagüeyes u otros medios. No cuentan con abastecimiento permanente de agua potable y mucho menos con servicio domiciliario, lo que impacta negativamente su alimentación y por ende su salud. En la actualidad el departamento de La Guajira, representado en niños de comunidades indígenas Wayuu, el más alto índice de muertes por desnutrición de Colombia. Y todos los factores aquí analizados influyen alta y negativamente en ello (Ruiz, 2017).

Con respecto a los servicios públicos domiciliarios, el Censo de 2005 realizado por el DANE provee información sobre el acceso al agua potable, la electricidad y los servicios de saneamiento. Del análisis de estas variables se desprende que existen fuertes desigualdades entre las áreas rurales y las urbanas. En particular, los hogares de las áreas urbanas muestran, en promedio, una alta cobertura (superior al 90\%) en el acceso al agua potable, la electricidad y los servicios de saneamiento. En contrapartida, en las áreas rurales los hogares presentan un acceso limitado a estos servicios (40,2\% de acceso al agua potable, 53,1\% de acceso a los servicios de saneamiento y $72,8 \%$ de acceso a la electricidad), lo que limita entre otros el uso de aparatos que se alimenten con energía eléctrica, que proveen bienestar y acceso a la información que circula en la localidad y en el mundo. Las brechas tecnológicas, comunicacionales y de acceso a servicios básicos, son evidentes.

La comunidad indígena Wayuu de Manzana enfrenta el impacto de condiciones socioeconómicas desfavorables. Se hace necesaria la inmediata acción del gobierno local y de los organismos nacionales para atender las dificultades que tienen. No se ve el impacto de la responsabilidad social de la Empresa que tiene a cargo la extracción del gas.

Los estudiantes después de cada visita consideran que la realidad que observaban superaba los datos que recogían; opinan que su condición de habitantes urbanos no les permitía dimensionar en su total complejidad lo que representaban cifras o datos numéricos sobre "deficiencia", "carencia", "acceso limitado" "debilidades en el servicio" con sus respectivos porcentajes. Construyeron conocimiento social sobre aspectos como aseo, servicio de energía, educación, servicios sanitarios, posesión y uso de tecnología. Este conocimiento fue utilizado 
por ellos para pensar y proponer como ejercicio académico unas mejores formas de solución a las condiciones de convivencia en que estaban las comunidades. Generaron representaciones gráficas con los que podían modelar la realidad (Aarón et al., 2016). La recolección de los datos y la observación directa fue valiosa, desde que les permitió relacionar el contexto con los conceptos y la realidad tecnológica y social.

\section{CONCLUSIONES}

A partir de los resultados obtenidos, se pueden indicar las siguientes conclusiones: 1) La comunidad indígena Wayuu de Manzana enfrenta condiciones socioeconómicas sustancialmente desfavorables en relación con el resto de la población, evidenciándose lo que históricamente han mostrado otros estudios como es la desigualdad de oportunidades y la discriminación de estos grupos minoritarios. Se hace necesario que los entes gubernamentales en su agenda prospectiva planeen políticas compensatorias y de nivelación de la igualdad de oportunidades y así tomar medidas específicas para luchar contra la desigualdad social, atendiendo de manera inmediata educación, lo sanitario, ambiental, así como el suministro de agua potable. 2) La caracterización de la comunidad indígena Wayuu de Manzana, se convirtió en una herramienta útil que ayuda a la comprensión de la comunidad como un sistema, donde se pueden apreciar factores que la afectan para lograr su desarrollo; el mal manejo sanitario deteriora sus condiciones ambientales, el agua, cuyo mayor abastecedor se da por la acción de la naturaleza, que unida a la condición desértica de la región y la ausencia de servicios públicos afecta su supervivencia. El alto analfabetismo y el bajo nivel educativo de la mayoría de los miembros de la comunidad, sumado a las débiles actividades económicas, generan una situación que propicia inequidad, desigualdad social y evidencia pobreza (Hall y Patrinos) y (Sen). 3) Los estudiantes participantes, observadores de la realidad, generaron puentes de comprensión sobre la importancia de contar con representaciones descriptivas y gráficas que constituyan de manera exacta la realidad, sin perder detalle. 4) A los estudiantes les sirvió la aproximación a la realidad desde sus sentidos, para construir representaciones modélicas, pasando del conocimiento tácito al explicito, sobre una comunidad indígena en condiciones de marginalidad. 5) La ingeniería social en este trabajo permitió desarrollar en los estudiantes del programa de Ingeniería de Sistemas habilidades para comprender la interacción entre problemas tecnológicos y sociales, la responsabilidad social y los procesos de transformación tecnológica.

\section{REFERENCIAS}

Aarón, M. A., The context: an element of analysis for teaching, doi: 10.14482/zp.25.9790, Zona Próxima, (25), 34-48 (2016)

Aaron, M, O. Castañeda y A.R. Ibarra, The Management and Construction of Knowledge as an Innovation Strategy for Collaborative Learning Through the Use and Creation of Learning Communities and Networks, The International Journal of Knowledge Management (IJKM) ISSN 1548-0666, elSSN 1548-0658, 38-48 (2014)

Aarón, M. A., P. E. Choles y A. D. Solano, Formative Process Representation of an Ethnic Education Institution through the Technique of Graphic and Descriptive Model Using Influence and Forresters Diagrams, Rev. Información Tecnológica, ISSN: 0718-0764, 27(3), 81-92 ( 2016)

Ander-Egg, E., Repensando la Investigación-Acción-Participativa, Grupo Editorial, 4ª Ed., 29-42, Lumen Humanitas (2003)

Bonilla-Castro, E., J. Hurtado y C. Jaramillo, La Investigación. Aproximaciones a la construcción del conocimiento científico, Colombia, Alfaomega (2009)

Carino, J., Indigenous Peoples, Human Rights and Poverty. In Tebtebba Foundation, Making the MDGs Relevant for Indigenous Peoples, Indigenous Perspectives, 6 (1) 28-46 Foundation (2005)

Chenail, R. J., Conducting Qualitative data Analysis: Qualitative data Analysis as a Metaphoric process, The Qualitative Report, 17(1), 248 (2012)

Cortés, F., Consideraciones sobre la Marginalidad, Marginación, Pobreza y Desigualdad en la distribución del ingreso, Papeles de Población, 8(31), 9-24 (2002)

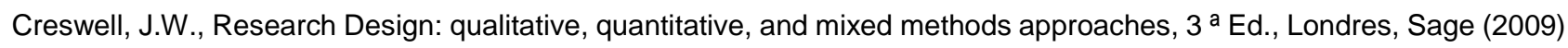

DANE, Departamento Administrativo Nacional de Estadística, Colombia, Censo General 2005, Bogotá (2006)

Desai, M., Poverty and Capability: Toward an Empirically Implementable measure, Frontera Norte, 6(1e), 11-30 (2017)

Dussán, C.P. y G.A. Rodríguez, Comunidades étnicas en Colombia: cultura y jurisprudencia, U. del Rosario (2005)

García, E., Introducción a la Cultura de Participación, Participación, Currículum y Educación Superior, México, Universidad del Centro de México y Grupo hacia una Ingeniería en Comunicación Social (2014)

Hall, G. y H. A. Patrinos, Pueblos Indígenas, Pobreza y Desarrollo Humano en América Latina, 1994-2004, Washington DC: Banco Mundial (2005)

IUCN, Inter-Commission Task Force on Indigenous Peoples. Indigenous Peoples and Sustainability, Cases and Actions, Utrecht, Países Bajos, International Books (1997)

Jaramillo, L. C. La ingeniería Social: Un desafío Investigativo, Revista Universidad Eafit, 104, $81-91$ (2012) 
Kennedy, A.M. y A. Parsons, Macro-social marketing and social engineering: a systems approach, Journal of Social Marketing, 2(1), 37-51 (2012)

López, E. J., Estudiar y construir la Cultura de Participación desde la interdisciplinariedad y la Ingeniería Social en el marco del quehacer científico en México, Razón y palabra, (90), 6 (2015)

López, N. y I. Sandoval, Métodos y Técnicas de investigación cualitativa y cuantitativa. Documento de Trabajo, Sistema de Universidad Virtual, Universidad de Guadalajara, 3-23 (2006)

Méndez, C., Metodología. Diseño y desarrollo del proceso de investigación, 3ª Ed., Bogotá, McGraw-Hill (2001)

Morín, E. y A. Sánchez, La ecología de la civilización técnica. De la noción de medio técnico a ecosistema social, Universitat de Valencia (1981)

Olivares, B., D. Lobo y otros tres autores, Socio-economic characteristics and methods of agricultural production of indigenous community Kashaama, Anzoategui, Venezuela, Rev. Fac. Agron. (LUZ) 34 (2), 187-215 (2017)

Ortiz, J., J. Miranda y otros tres autores, Seroprevalencia de Rickettsia sp. En indígenas Wayuü de la Guajira y Kankuamos del Cesar, Colombia, Infectio, 19(1), 18-23 (2015)

Premauer, J.M. y F. Berkes, A pluralistic approach to protected area governance: indigenous peoples and Makuira National Park, Colombia, Ethnobiology and Conservation, 4 (2015)

Psacharopoulos, G. y H.A. Patrinos, Indigenous people and poverty in Latin America: An empirical analysis, Banco Mundial, Washington (1994)

Rodríguez, A., Breve reseña de los derechos y de la legislación sobre comunidades étnicas en Colombia, Bogotá, Universidad del Rosario (2007)

Ruiz, N. J. R., Las mortalidades por desnutrición, una realidad que violenta los derechos humanos, Colombia 2003-2012, Anais, 1-29 (2017)

Sánchez, A., Introducción: ¿qué es Caracterizar? Medellín, Fundación Universitaria Católica del Norte (2010)

Sea, C., Colombia: Makuira, the cosmological centre of origin for the Wayúu people, Protected Landscapes and Wild Biodiversity, 3, 53 (2012)

Sen A., The Political Economy of Targeting. Annual Bank Conference on Development Economics, World Bank (1992)

UNESCO, Convención para la salvaguarda del patrimonio cultural inmaterial, París (2003)

UNICEF, Los pueblos indígenas en Colombia, Derechos, políticas y desafios. Bogotá, Colombia: UNICEF (2007) 
